\title{
Quantitative Cortical Mapping of Fractional Anisotropy in Developing Rat Brains
}

\author{
Hao Huang, ${ }^{1,3}$ Akria Yamamoto, ${ }^{1,4}$ Mir Ahamed Hossain, ${ }^{2}$ Laurent Younes, ${ }^{5}$ and Susumu Mori ${ }^{1,6}$ \\ Departments of ${ }^{1}$ Radiology and ${ }^{2}$ Neurology, School of Medicine, Johns Hopkins University, Baltimore, Maryland 21205 , ${ }^{3}$ Advanced Imaging Research \\ Center, University of Texas Southwestern Medical Center, Dallas, Texas 75390, ${ }^{4}$ Department of Diagnostic Imaging and Nuclear Medicine, Kyoto \\ University, Kyoto 6048845, Japan, ${ }^{5}$ Center of Imaging Science, Johns Hopkins University, Baltimore, Maryland 21218, and ${ }^{6} \mathrm{~F}$. M. Kirby Research Center for \\ Functional Brain Imaging, Kennedy Krieger Institute, Baltimore, Maryland 21205
}

Cortical development is associated with a series of events that involve axon and dendrite growth and synaptic formation. Although these developmental processes have been investigated in detail with histology, three-dimensional and quantitative imaging methods for rodent brains may be useful for genetic and pharmacological studies in which cortical developmental abnormalities are suspected. It has been shown that diffusion tensor imaging (DTI) can delineate the columnar organization of the fetal and early neonatal cortex based on a high degree of diffusion anisotropy along the columnar structures. This anisotropy is known to decrease during brain development. In this study, we applied DTI to developing rat brains at five developmental stages, postnatal days $0,3,7,11$ and 19 , and used diffusion anisotropy as an index to characterize the structural change. Statistical analysis reveals four distinctive cortical areas that demonstrate a characteristic time course of anisotropy loss. This method may provide a means to delineate specific cortical areas and a quantitative method to detect abnormalities in cortical development in rodent pathological models.

Key words: rat; brain; development; magnetic resonance imaging; diffusion; dendrite; cortex

\section{Introduction}

Mammalian brain development is a complicated process. The cortical neurons are generated in the subventricular zone in the fetal stage and migrate toward the cortical plate after the radial scaffold formed by glial cells (radial glia). During the fetal period, axons from and to the cortical plate also start to form, which tend to align the radial glia in the early phase of development. These radial structures create the columnar organization in the fetal cortex (Sidman and Rakic, 1973; Rakic, 2002). In the postnatal period, a series of events involving dendrite and axon growth and synaptic formation occur in the cortex (for review, see McAllister, 2000). These biological events and their molecular mechanisms have been the targets of extensive research in the past decade, for which histology played a vital role in anatomical characterization.

Although histology-based techniques remain the gold standard method for anatomical characterization, phenotype or pharmacological studies would be greatly enhanced by threedimensional (3D) imaging methods that can efficiently and quantitatively monitor brain development processes and detect abnormalities. In the past, high-resolution magnetic resonance imaging (MRI) has been used to examine brain morphology, myelination, and axonal growth (Johnson et al., 1997; Ahrens et

Received July 13, 2007; revised Dec. 11, 2007; accepted Dec. 13, 2007.

This work was supported by National Institutes of Health Grants R01EB003543 and R01ES012665.

Correspondence should be addressed to Dr. Susumu Mori, The Russell H. Morgan Department of Radiology and Radiological Science, The Johns Hopkins University School of Medicine, 217 Traylor Building, 720 Rutland Avenue, Baltimore, MD 21205.E-mail: susumu@mri.jhu.edu.

DOI:10.1523/JNEUROSCI.3194-07.2008

Copyright $\odot 2008$ Society for Neuroscience $\quad$ 0270-6474/08/281427-07\$15.00/0 al., 1998; Jacobs et al., 1999; Benveniste and Blackband, 2002). Diffusion tensor imaging (DTI), one type of MRI, is especially useful for the delineation of fetal/embryonic and neonatal brain anatomy (Huppi et al., 1998; Neil et al., 1998; Mori et al., 2001; McKinstry et al., 2002; Mukherjee et al., 2002; Maas et al., 2004; Partridge et al., 2004; Schneider et al., 2004; Hermoye et al., 2005; Huang et al., 2006). DTI is sensitive to anisotropic diffusion of water molecules and can be used to depict the existence and the orientations of the ordered structures (Moseley et al., 1990; Basser et al., 1994). Fractional anisotropy (FA) is an index of the extent of aligned structures, and its value ranges from zero to one. For isotropic water diffusion, the water displacements are equal in every direction and the FA value is zero, whereas diffusion in ordered structures has a higher FA.

In the fetal and neonatal stages, columnar structures dominate the mammalian cortex, resulting in relatively high FA values (Thornton et al., 1997; Neil et al., 1998; Mori et al., 2001; McKinstry et al., 2002; Neil et al., 2002). In the early postnatal period, FA of the cortex lessens dramatically, which has been attributed to the loss of the radial glia and to increased dendritic density (Neil et al., 1998).

In this study, we acquired high-resolution and high signal-tonoise ratio (SNR) DTI data from developing rat brains and monitored the four-dimensional spatiotemporal (three dimensions for geometrical mapping and one dimension of the time axis) maturation process of the cortex. In the rat brain, dendritic growth occurs mostly at approximately postnatal day $1(\mathrm{P} 1)$ to P7, followed by synapse formation by P7-P14 (Ince-Dunn et al., 2006). Five developmental time points, P0, P3, P7, P11, and P19, were chosen to cover this developmental period. The FA values 
were measured in various cortical areas as a quantitative marker of anatomical changes.

\section{Materials and Methods \\ Animal subjects}

Time-pregnant (gestational day 16-18) female Sprague Dawley rats from Charles River Breeding Laboratories (Wilmington, MA) were used for the experiments. All animals were housed in individual cages with a $12 \mathrm{~h} \mathrm{light/dark} \mathrm{cycle} \mathrm{with} \mathrm{access} \mathrm{to} \mathrm{food} \mathrm{and} \mathrm{water} \mathrm{ad} \mathrm{libitum.} \mathrm{After} \mathrm{the}$ pups were born, the number of pups was culled to eight pups per dam. Rat pups were killed by decapitation at five time points at the ages of $\mathrm{P} 0$, P3, P7, P11, and P19 and were examined. Their heads were fixed with $4 \%$ paraformaldehyde in PBS and remained in fixation solution for 2 weeks. Before MR imaging, we placed rat heads in PBS for $>48 \mathrm{~h}$ and transferred them into custom-made, MR-compatible tubes. The rat heads were bathed with fomblin (Fomblin Profludropolyether; Ausimont, Thorofare, NJ) during scanning. At each time point, two pups from different litters were scanned. All procedures involving animals were performed in accordance with the National Institutes of Health Guide for the Care and Use of Laboratory Animals and were approved by The Johns Hopkins University Animal Care and Use Committee.

\section{MRI data acquisition}

A Bruker 9.4 T scanner was used for data acquisition. Depending on the size of the specimens, volume coils with different inner diameters (15-25 $\mathrm{mm}$ ) were used for transmitter and receiver. A 3D multiple spin echo sequence with six echoes was used to acquire diffusion-weighted images (DWIs) (Zhang et al., 2002, 2003, 2005). From the six echoes, six individual $3 \mathrm{D}$ volume images were obtained, which were averaged to enhance the SNR. The parameters for DWI were as follows: field of view, $10-18 / 10-18 / 10-18 \mathrm{~mm}$; $3 \mathrm{D}$ imaging matrix, $128 \times 80 \times 72$ (zero filled to $128 \times 128 \times 128$ ); echo time, $34 \mathrm{~ms}$; repetition time, $700 \mathrm{~ms}$; six independent diffusion-weighted directions with a $b$ value of $1000 \mathrm{~s} / \mathrm{mm}^{2}$; and two additional images with minimal diffusion weighting ( $b$ of 50 $\mathrm{s} / \mathrm{mm}^{2}$ ). The pixel size after zero-filling for DTI images was $80-150 \mu \mathrm{m}$. Two signal averages with a phase cycling were used. For each specimen, the DTI acquisition time was $\sim 20 \mathrm{~h}$.

\section{Tensor fitting and FA calculation}

Six elements of the $3 \times 3$ diffusion tensor were determined by multivariate least-squares fitting of raw DWIs. The tensor was diagonalized to obtain three eigenvalues $\left(\lambda_{1-3}\right)$ and eigenvectors $\left(\nu_{1-3}\right)$. FA was calculated with the following equation:

$$
\mathrm{FA}=\frac{\sqrt{\left(\lambda_{1}-\lambda_{2}\right)^{2}+\left(\lambda_{1}-\lambda_{3}\right)^{2}+\left(\lambda_{2}-\lambda_{3}\right)^{2}}}{\sqrt{2} \sqrt{\lambda_{1}^{2}+\lambda_{2}^{2}+\lambda_{3}^{2}}} .
$$

The eigenvector associated with the largest eigenvalue $\left(\nu_{1}\right)$ was used as an indicator of fiber orientation. For color-coded orientation maps, red (R), green $(G)$, and blue (B) colors were assigned to left-right, anteriorposterior, and superior-inferior orientations, respectively. For the color presentation, 24-bit color was used, in which each RGB color had 8-bit $(0-255)$ intensity levels. The unit vector $\nu_{1}\left(=\nu_{1 x}, \nu_{1 y}, \nu_{1 z}\right)$ always fulfills the condition $\nu_{1 x}{ }^{2}+\nu_{1 y}{ }^{2}+\nu_{1 z}{ }^{2}=1$. Intensity values of $\nu_{1 x}{ }^{2} \times 255$, $\nu_{1 y}{ }^{2} \times 255$, and $\nu_{1 z}{ }^{2} \times 255$ were assigned to the R, G, and B channels, respectively. To suppress orientation information in isotropic brain regions, the 24-bit color value was multiplied by FA, which was scaled from 0 to 1 . Average diffusion-weighted images (aDWIs) were obtained by adding all diffusion-weighted images.

\section{Sampling point placement and cortical structure labeling}

As shown in Figure 1, there are few prominent anatomical landmarks on the rat cortical surface that would facilitate placing measurement points at the equivalent anatomical locations throughout brain development. In this study, we identified corresponding coronal slices based on proportions related to the cortical length in the caudal-rostral axis; the caudal and rostral edges of the cerebral cortex was denoted as $L$. Then coronal slices were defined at $\frac{L}{5}, \frac{2 L}{5}, \frac{3 L}{5}$, and $\frac{3 L}{4}$, as shown in Figure 1. Sixteen

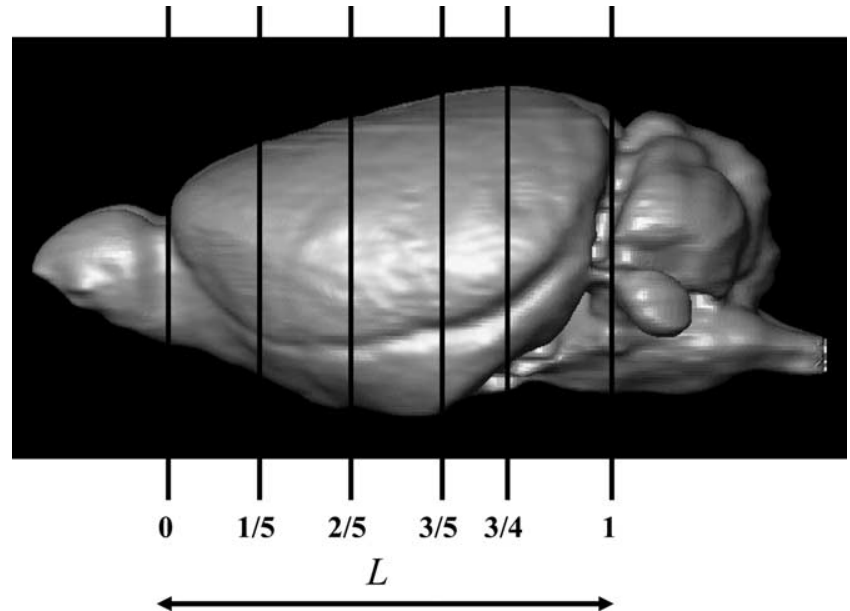

Figure 1. A three-dimensional view of a P19 rat brain and locations of coronal slices for cortical FA measurements.

measurement points for FA values were first defined in the P19 brain using the Paxinos atlas (Paxinos and Watson, 2005) as a guide to cover all the major functional areas (Fig. 2), namely, the somatosensory, motor, cingulate, prelimbic, insula, visual, and auditory cortices. The Paxinos atlas could not be used for younger brains because of substantial anatomical differences from the atlas based on an adult brain. The measurement points defined on the P19 brain were transferred to the younger brains based on the angles from the midline. The angles were as follows: $5^{\circ}, 60^{\circ}$, $100^{\circ}\left(\frac{L}{5}\right.$ slice $) ; 0^{\circ}, 10^{\circ}, 40^{\circ}, 70^{\circ}, 100^{\circ}\left(\frac{2 L}{5}\right.$ slice $) ; 30^{\circ}, 60^{\circ}\left(\frac{3 L}{5}\right.$ slice $)$; and $5^{\circ}$, $30^{\circ}, 60^{\circ}$ ( $\frac{3 \mathrm{~L}}{4}$ slice $)$. The sampling points on the medial cortical areas, which were for the cingulate and the prelimbic cortices, were manually placed.

\section{Cortical FA mapping and measurement}

FA mapping of the cortical outer surface. The entire brain was defined using the intensity thresholding of aDWIs. Then, triangular meshes were created from isosurface, which defines the brain surface. To visualize the FA values of the cortex, the defined brain surface was shrunk toward the inside of the brain by two pixels. On each triangular mesh, an FA value was assigned by reading the FA value of the closest pixel toward the inside of the brain. The visualization of these triangular meshes with FA values was accomplished using the Amira software (TGS, San Diego, CA).

FA measurement at fixed sampling points. For the sixteen measurement points described above, detailed time courses were studied. At each sampling point, a small line segment was defined from the outer surface to the nearby white matter in the two-dimensional coronal slices. The white matter-gray matter boundaries were defined by FA and by changes in fiber orientations (color). Because of the almost $90^{\circ}$ difference in the axon orientations between the white matter and the cortex, there is a low-FA band between the two structures (Fig. 2). At each measurement point, a line that connects the surface and the nearest white matter was manually defined. Because of the gradient of FA values from the surface of the cortex toward the white matter (Sizonenko et al., 2007), the line was segmented into three equidistant sections as shown in Figure $2 a$, and FA was measured separately for each segment.

\section{Statistical analysis}

Theory. Statistical analysis was performed on the FA values of the outer segments, in which significant changes occurred during development. The parameters $\mathrm{FA}(1, k, t)$ and $\mathrm{FA}(2, k, t)$ were defined as the measurement of FA on two rat brain cortices, where $t$ was the time point and $k$ was the considered brain region. There were $T$ time points and $M$ cortical regions, where $T=5$ and $M=16$. The null hypothesis was at fixed time $t$, and all $2 M$ measurements were statistically indistinguishable. For fixed time $t, R k(i, k, t)$ was defined as the rank of $\mathrm{FA}(i, k, t)$ in the sequence of $2 N$ numbers $\operatorname{FA}(1,1, t)$, 


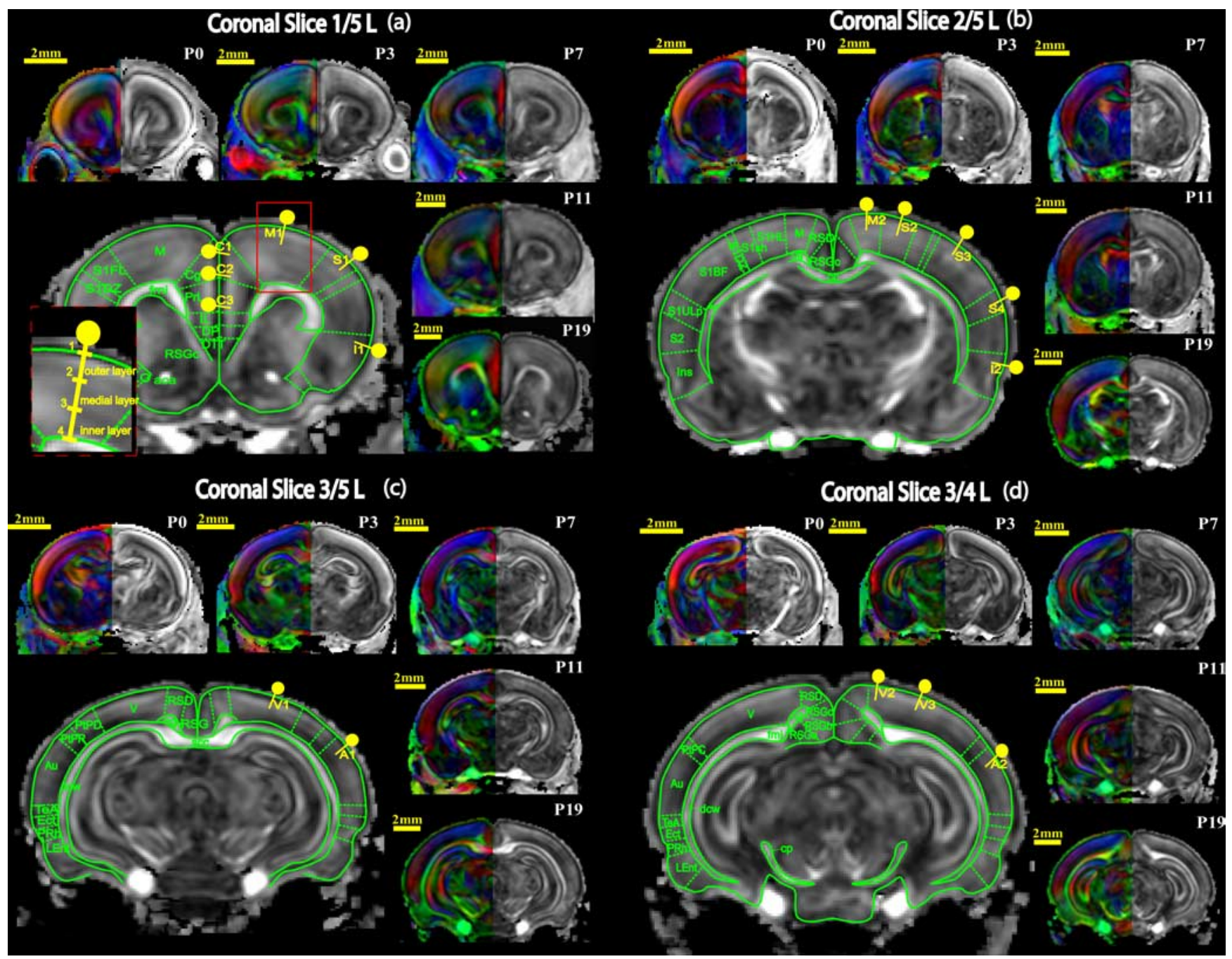

Figure 2. FA maps and color-coded orientation maps of developing rat brains at $1 / 5 \mathrm{~L}(\boldsymbol{a}), 2 / 5 \mathrm{~L}(\boldsymbol{b}), 3 / 5 \mathrm{~L}(\boldsymbol{c})$, and $3 / 4 \mathrm{~L}$ (d) slices defined in Figure 1 . FA and color-coded maps are shown for the right and left hemispheres of the P0, P3, P7, P11, and P19 rat brains. The FA map of a P19 brain is enlarged, and the anatomical definition from the Paxinos atlas is superimposed. Yellow dots indicate the locations of sampling points for cortical FA measurements. The inset in $\boldsymbol{a}$ illustrates the three segments defined in this study for FA measurements. For the color-coded maps, red, green, and blue colors indicate structures aligning along left-right, anterior-posterior, and superior-inferior orientations, respectively.

$\mathrm{FA}(2,1, t), \ldots, \mathrm{FA}(1, N, t), \mathrm{FA}(2, N, t)$, where $N$ values are part of $M$ cortical regions. The Kruskal-Wallis statistics were defined as follows:

$$
K=\sum_{t=1}^{T} \sum_{k=1}^{N}(R k(1, k, t)+R k(2, k, t)-2 N-1)^{2} .
$$

We sampled random permutations of the data to generate a $p$ value for the rejection of the null hypothesis. We calculated the $K_{M C}$ value of each randomly generated sequence. Because, under the null hypothesis, all sequences of ranks have the same probability, a $p$ value for the observed $K$ can be computed as the proportion of Monte Carlo samples that are above it. More precisely, if there were $N_{M C}$ Monte Carlo simulations and $N_{\text {less } K}$ was the number of simulations in which $K_{M C}$ values were less than the observed $K$ from cortical FA data, we compute the $p$ values for $K$ as follows:

$$
p=1-\frac{N_{\text {less } K}+0.5}{N_{M C}} .
$$

Comparison with average. We calculated the averaged FA from the sixteen measurement points and compared it with the individual FA measurements. In this case, only two datasets were compared: the averaged and individual FA. The parameters for $K$ value calculation in Equation 2 were $N=2$ and $T=5$.
Intragroup comparison. From the atlas, measurement points from the same cortical region were grouped together. Intragroup comparison was performed for the insula, cingulate, auditory, visual, somatosensory, and motor cortical regions. Depending on how many sampling points we had for each cortical region, the parameters for $K$ value calculation in Equation 2 were $N=2-4$ and $T=5$.

Intergroup comparison. Based on the cortical parcellation by superimposition of the Paxinos atlas (Fig. 2), we calculated the average FA of each parcellation and conducted an intergroup comparison based on the average FAs. The parameters for $K$ value calculation in Equation 2 were $N=2$ and $T=5$.

Multiple-comparison correction. Bonferroni's correction (Bonferroni, 1936; Shaffer, 1995) was used to avoid the spurious positives when rejecting the null hypothesis. Instead of using threshold 0.05 for $p$ values, $0.05 / N_{\text {area }}$ was used to lower the threshold and account for the number of comparisons being performed. The threshold correction was only applied to intergroup comparison in which heterogeneity of seven areas was tested.

\section{Results}

Fractional anisotropy and color-coded map of developing rat brains

Figure $2 a-d$ shows the coronal slices of FA maps and color maps at the five different time points (P0-P19) and at the four observation slices indicated in Figure 1. In the images from P19, corti- 
cal parcellation from the Paxinos atlas (Paxinos and Watson, 2005) is overlaid and the sixteen measurement points are indicated. In these images, loss of cortical FA during P0-P11 can be visually appreciated. At P0, FA of the outer cortical layer is visibly higher than that of the white matter. During P11-P19, this cortex-white matter contrast inverts; the FA of the white matter becomes higher than that of the cortex.

\section{Fractional anisotropy mapping on the cortical outer surface}

Figure 3 shows the spatiotemporal FA variation of the cortical surface. From P0 to P11, the FA of the cortex undergoes a dramatic decrease, with the highest value $(\mathrm{FA}=0.7-1.0)$ at $\mathrm{P} 0$, dropping to $0.3-0.4$ in most cortical areas at P7. There is an overall anterior-posterior gradient associated with the FA decrease. After P11, there is no substantial change in the FA (0.20.3 ). The medioanterior regions with relatively low FA at P0 correspond to motor (M1) and sensory (S1) cortices. The lateroposterior regions with relatively high FA at a later stage (P7) correspond to visual (V3) and auditory (A2) cortices according to the Paxinos atlas (Fig. 2).

\section{Quantitative characterization of fractional anisotropy at sampling points and three cortical layers}

To quantitatively analyze the spatiotemporal changes of cortical FA, regional FA measurements were performed at the sixteen locations shown in Figures 2 and 3 using ten rat brain samples. In Figure 4, the measurement points are classified based on their cortical areas, and the quantitative measurement results are shown. Figure 4 shows the FA values of the outer and the inner sections of the cortex (for the definition of the cortical divisions, see Fig. 2a). The FA of the inner sections are within a small range (mostly $0.15-0.25$ ), regardless of the locations and ages, which is expected, because the boundary (and thus the innermost layer) is defined by low FA.

The age-dependent loss of the FA of the cortex can be clearly appreciated in the outer segment, and each cortical area has a unique time course. Among all cortices measured in this study, the cingulate ( $\mathrm{C} 1$ and $\mathrm{C} 2$ ) and prelimbic $(\mathrm{C} 3)$ FA showed the least time dependency (Fig. 4a). Although they tend to have lower FA at P0, they eventually become the cortices with the highest FA, which can be clearly appreciated in Figure $2 a$. The FA of the insula is characterized by early loss of FA (Fig. $4 b$ ). The FA of most somatosensory and motor areas follows the average time course closely (Fig. $4 c, d$ ). On the contrary, the visual and auditory areas are characterized by high FA at P0-P7 (Fig. 4e,f), as can be visually appreciated in Figure 3. In Figure 5, diffusivity, radial $\left(\lambda_{1}\right)$ and tangential $\left[\left(\lambda_{2}+\lambda_{3}\right) / 2\right]$ to the pial surface at two representative locations (prelimbic and somatosensory), is shown. In both cortices, the tangential diffusivity remains stable throughout postnatal development, whereas the radial diffusivity of the somatosensory cortex decreases rapidly in the first 2 weeks, in agreement with previous reports of human neonatal studies (Deipolyi et al., 2005).

\section{Statistical analysis}

Statistical analysis was performed to characterize the time course of the cortical areas, which are statistically different from the average (Table 1) and statistically different from each other (Table 2). As shown in Table 1, the ten sampling points at the cingulate, prelimbic, insula, visual, and auditory cortices are statistically different from the average FA $(p<0.05)$. The six points at the somatosensory and motor cortex are all similar to the average FA, except for the barrel field (S3), which has a slightly higher FA throughout development (Fig. 4c). In Table 1, the intragroup comparison is also shown. The time courses of the same cortical areas are statistically similar. The FA values measured at different locations, but which belong to the same cortical areas, were thus averaged to perform the intergroup statistical analysis.

Table 2 shows the intergroup statistical analysis result based on the averaged FA values within the cortical areas. From the statistical analysis, there appear to be four distinctive groups: the cingulate/prelimbic, the insula, the visual/auditory; and the sensory/motor.

\section{Discussion}

Although the anatomy of neurons and their dendrites has been well documented qualitatively in the past (Ramon y Cajal, 1909; Ramon-Moliner, 1962), our knowledge about regional variation across cortical areas during brain development is scarce because histology-based studies have focused on only one region of the brain at a time (Jacobs et al., 2001). Therefore, a method for quantitative and spatial measurements of dendrite density would be extremely useful.

In this study, we characterized the spatiotemporal changes in the diffusion anisotropy of the rat cortex in the early neonatal period. The observed changes in the water diffusion property reflect underlying anatomical changes. In fetal/embryonic and newborn brains, it has been shown that the water diffusion is highly anisotropic and its orientation is perpendicular to the brain surface, representing the cortical columns (Thornton et al., 1997; Neil et al., 1998; Baratti et al., 1999; Mori et al., 2001; McKinstry et al., 2002; Maas et al., 2004; Huang et al., 2006; Sizonenko et al., 2007). The dramatic loss of anisotropy in P0-P7 indicates that water diffusion becomes more random in those stages. In human studies, McKinstry et al. (2002) reported that the time course of anisotropy diminishment coincides with the period of "cortical maturation" (Sidman and Rakic, 1982) and attributed the decrease to the growth of dendrites, as well as formation of cell processes and their connections, to the arrival of local circuit neurons and their differentiation, and to the disappearance of the radial glia (Sidman and Rakic, 1973; Kostovic and Rakic, 1990). In rat brains, axonal and dendritic branching in the 
(a)

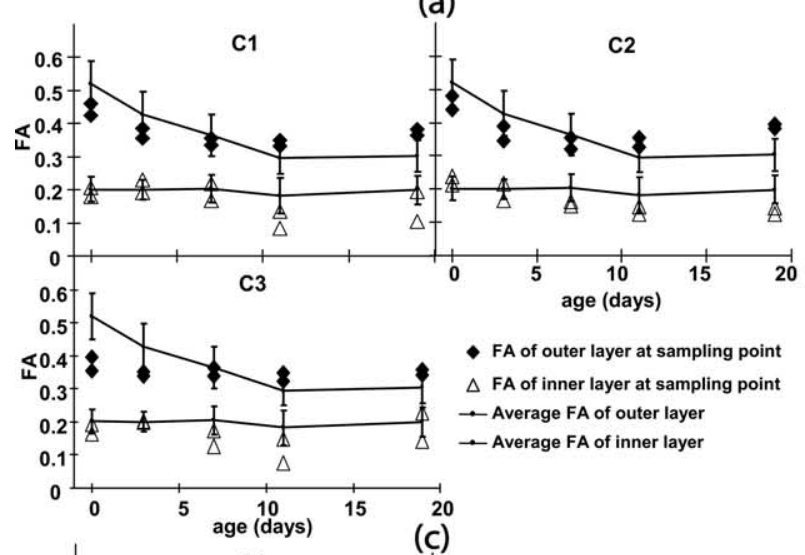

s1

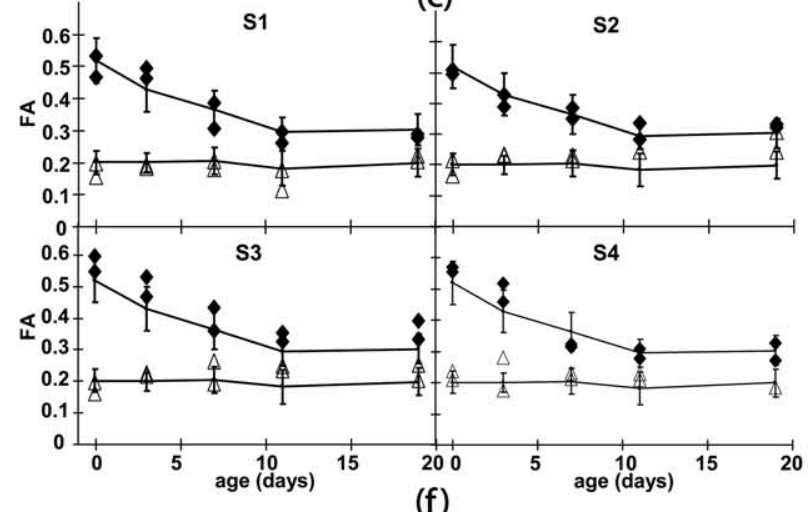

A1

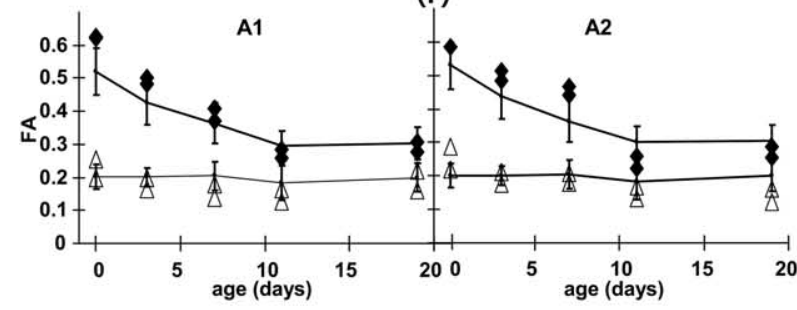

(b)

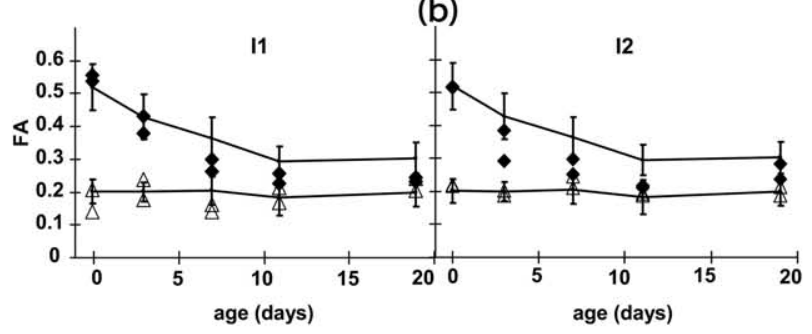

(d)

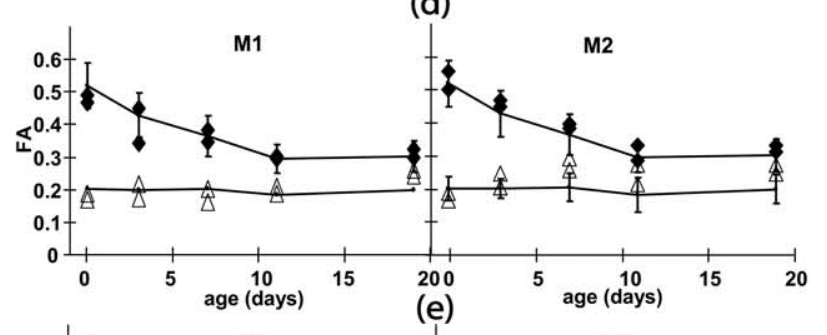

v1

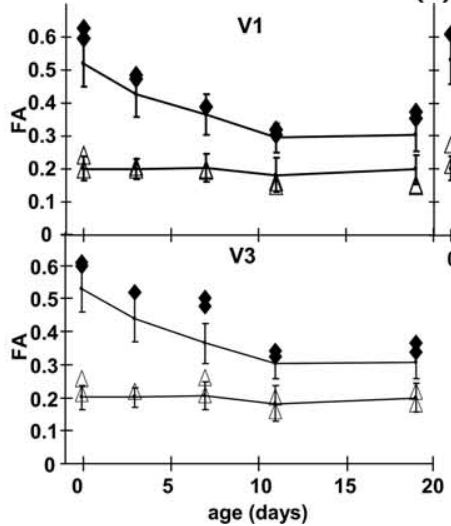

Figure 4. FA measurement results from various cortical regions during the $\mathrm{PO}-\mathrm{P} 19$ period. The FA values averaged over all sixteen measurement points are shown as reference curves, with error bars indicating SDs. $\boldsymbol{a}$, The cingulate (C1, C2) and the prelimbic (C3) cortices; $\boldsymbol{b}$, the insula cortex (I1, I2); $\boldsymbol{c}$, the somatosensory cortex (S1-S4); $\boldsymbol{d}$, the motor cortex (M1, M2); $\boldsymbol{e}$, the visual cortex (V1-V3); and $\boldsymbol{f}$, the auditory cortex (A1, A2).
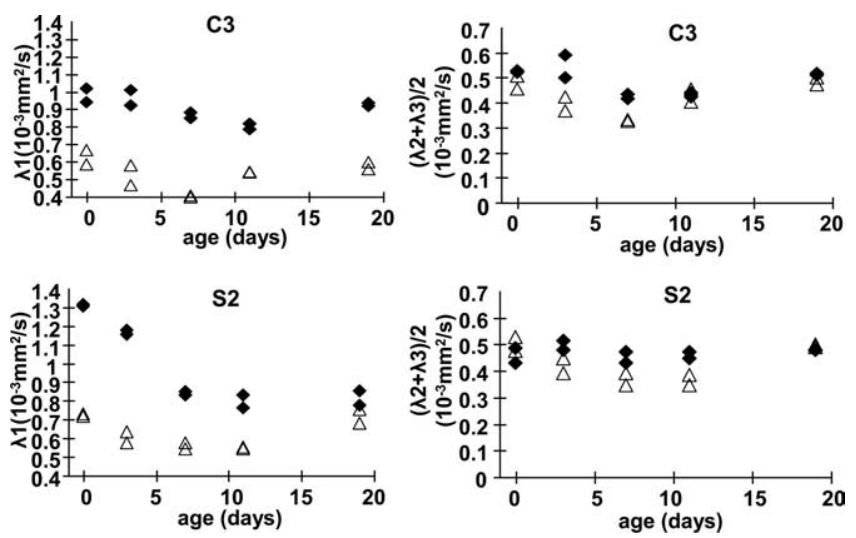

Figure 5. Time-dependent changes of the radial $\left(\lambda_{1}\right)$ and tangential $\left[\left(\lambda_{2}+\lambda_{3}\right) / 2\right]$ diffusivity of the prelimbic (C3) and somatosensory (S2) cortices.

cortex begins at approximately P1-P7, and the peak of synapse formation occurs at approximately P7-P14, whereas dendrite growth extends to the third week (Miller, 1981; Miller and Peters, 1981; Harris et al., 1992; Wong and Ghosh, 2002; Zhang, 2004).
This temporal course of dendritic maturation mostly matches the anisotropy change observed in this paper, although the FA decrease reaches a plateau after the second week at approximately $\mathrm{FA}=0.3$ (Fig. 4), which could suggest the influence of cortical maturation processes other than dendritic growth.

Using the sixteen measurement points, we identified four cortical areas with characteristic time courses: the cingulate and prelimbic cortices are characterized by low time dependency, the insula by early loss of FA, and the visual/auditory cortices by slower loss with respect to motor/sensory cortices. Human studies revealed similar results. Deipolyi et al. (2005) also found evidence of regional heterogeneity in human cortical development from the FA measurement of the superior frontal, superior occipital, precentral, and postcentral gyri.

Dendrite maturation studies (McAllister, 2000; Whitford et al., 2002; Libersat and Duch, 2004) have suggested that the morphogenesis of dendritic trees is regulated by innate genetic factors, neuronal activity, and external molecular cues. During development, neuronal activity induced by the afferent stimulation of the visual and auditory system occurs later than that of the somatosensory system. Our findings about FA, showing a delayed 
Table 1. $p$ values from statistical analysis of intracortical and intercortical differences in $F A$ values

\begin{tabular}{|c|c|c|c|c|c|c|c|c|c|c|c|c|c|c|c|c|}
\hline \multirow[b]{2}{*}{ Sampling Points } & \multicolumn{3}{|c|}{$\begin{array}{l}\text { Cingulate and prelimbic } \\
\text { cortices }\end{array}$} & \multicolumn{2}{|l|}{ Insula } & \multicolumn{3}{|c|}{ Visual cortex } & \multicolumn{2}{|c|}{ Auditory cortex } & \multicolumn{4}{|c|}{ Somatosensory cortex } & \multicolumn{2}{|c|}{ Motor cortex } \\
\hline & $\mathrm{C1}$ & $\mathrm{C} 2$ & C3 & 11 & 12 & V1 & V2 & V3 & A1 & $A 2$ & $S 1$ & S2 & S3 & $\$ 4$ & M1 & M2 \\
\hline $\begin{array}{l}p \text { value }^{a} \\
p \text { value }^{b}\end{array}$ & $\begin{array}{l}\mathbf{0 . 0 0 4} \\
0.725\end{array}$ & 0.004 & 0.026 & $\begin{array}{l}\mathbf{0 . 0 2 6} \\
0.254\end{array}$ & 0.026 & $\begin{array}{l}\mathbf{0 . 0 0 4} \\
0.405\end{array}$ & 0.004 & 0.004 & $\begin{array}{l}\mathbf{0 . 0 4 7} \\
0.367\end{array}$ & 0.004 & $\begin{array}{l}0.501 \\
0.053\end{array}$ & 0.501 & 0.024 & 0.205 & $\begin{array}{l}0.756 \\
0.501\end{array}$ & 0.205 \\
\hline
\end{tabular}

${ }^{a}$ Compared with the time course averaged from all measurement points. The $p$ values smaller than the threshold 0.05 are in bold.

${ }^{b}$ Compared within each cortical area.

Table 2. $p$ values from statistical analysis of FA values among different cortical areas

\begin{tabular}{|c|c|c|c|c|c|}
\hline$C$ & 1 & V & $A$ & $S$ & $M$ \\
\hline C & 0.048 & 0.004 & 0.004 & 0.027 & 0.004 \\
\hline I & & 0.004 & 0.004 & 0.004 & 0.004 \\
\hline V & & & 0.501 & 0.027 & 0.004 \\
\hline A & & & & 0.027 & 0.004 \\
\hline$S$ & & & & & 0.376 \\
\hline
\end{tabular}

C, Cingulate and prelimbic cortex; I, insula; V, visual cortex; A, auditory cortex; S, somatosensory cortex; M, motor cortex. The $p$ values smaller than the Bonferroni's-correction threshold $(0.05 / 7=0.0071)$ are in bold.

FA decrease for auditory and visual areas (Fig. 4e,f), may be related to the neuronal activity timeline of these systems.

Because of the lack of comprehensive histology-based studies of the spatial profile of the cortical maturation, it is difficult to further validate the correlation between spatiotemporal changes in FA and a specific cortical maturation process, such as dendrite growth. Because MRI techniques simply detect signals from water molecules, it is usually not possible to expect perfect correlation between changes in the MRI signal and a specific biological event. Therefore, the mapping of the cortical FA could only implicate the degree of complexity of the cortical anatomy, and the FA loss during $\mathrm{P} 0-\mathrm{P} 11$ should be considered an indirect parameter of dendrite growth. Nonetheless, because of its ability to detect changes in anatomical alignment quantitatively and three dimensionally, DTIs could be a useful tool to characterize normal growth, detect abnormal cortical regions and timings, and direct subsequent histology-based studies, thereby increasing the efficiency and, potentially, the sensitivity of histology studies.

In this study, all results were based on ex vivo samples. Because in vivo mouse DTIs have been successfully performed recently (Song et al., 2002, 2004), it is of great interest to know whether our findings are compatible with in vivo studies. Although we do not have data directly comparing ex vivo and in vivo results from the same animals, past studies suggest that FA is well preserved in postmortem samples (Sun et al., 2003, 2005). Our ex vivo results also indicate cortical FA of $\sim 0.3$ in P19 animals, which is comparable with in vivo results (Zhang et al., 2007).

\section{Conclusion}

In this study, we applied DT-MRI technology to study the development of the rat brain cortex in the neonatal period. Fourdimensional spatiotemporal FA mapping of the cortex reflects an increase in structural complexity during development. There is rapid decrease of FA in the first week of age and the spatial progression from the anterior to the posterior regions of the brain. Statistical analysis reveals four distinctive regions with different time courses: the cingulate/prelimbic, the insula, the visual/auditory, and the sensory/motor cortices. This method may provide a means to delineate specific cortical areas and a quantitative method to detect abnormalities in cortical development in rat pathological models.

\section{References}

Ahrens ET, Laidlaw DH, Readhead C, Brosnan CF, Fraser SE, Jacobs RE (1998) MR microscopy of transgenic mice that spontaneously acquire experimental allergic encephalomyelitis. Magn Reson Med 40:119-132.

Baratti C, Barnett A, Pierpaoli C (1999) Comparative MR imaging study of brain maturation in kittens with $\mathrm{t} 1, \mathrm{t} 2$, and the trace of the diffusion tensor. Radiology 210:133-142.

Basser PJ, Mattiello J, Le Bihan D (1994) MR diffusion tensor spectroscopy and imaging. Biophys J 66:259-267.

Benveniste H, Blackband S (2002) MR microscopy and high resolution small animal MRI: applications in neuroscience research. Prog Neurobiol 67:393-420.

Bonferroni CE (1936) Teoria statistica delle classi e calcolo delle probabilita. Pubblicazioni del R Istituto Superiore di Scienze Economiche e Commerciali di Firenze 8:3-62.

Deipolyi AR, Mukherjee P, Gill K, Henry RG, Partridge SC, Veeraraghavan S, Jin H, Lu Y, Miller SP, Ferriero DM, Vigneron DB, Barkovich J (2005) Comparing microstructural and macrostructural development of the cerebral cortex in premature newborns: Diffusion tensor imaging versus cortical gyration. NeuroImage 27:579-586.

Harris KM, Jensen FE, Tsao B (1992) Three-dimensional structure of dendritic spines and synapses in rat hippocampus (CA1) at postnatal day 15 and adult ages: implications for the maturation of synaptic physiology and long-term potentiation. J Neurosci 12:2685-2705.

Hermoye L, Saint-Martin C, Cosnard G, Lee SK, Kim J, Nassogne MC, Menten R, Clapuyt P, Donohue PK, Hua K, Wakana S, Jiang H, van Zijl PC, Mori S (2005) Pediatric diffusion tensor imaging: normal database and observation of the white matter maturation in early childhood. NeuroImage 29:493-504.

Huang H, Zhang J, Wakana S, Zhang W, Ren T, Richards LJ, Yarowsky P, Donohue P, Graham E, van Zijl PC, Mori S (2006) White and gray matter development in human fetal, newborn and pediatric brains. NeuroImage 33:27-38.

Huppi P, Maier S, Peled S, Zientara G, Barnes P, Jolesz F, Volpe J (1998) Microstructural development of human newborn cerebral white matter assessed in vivo by diffusion tensor magnetic resonance imaging. Pediatr Res 44:584-590.

Ince-Dunn G, Hall BJ, Hu S, Ripley B, Huganir RL, Olson JM, Tapscott SJ, Ghosh A (2006) Regulation of thalamocortical patterning and synaptic maturation by neuroD2. Neuron 49:683-695.

Jacobs B, Schall M, Prather M, Kapler E, Driscoll L, Baca S, Jacobs J, Ford K, Wainwright M, Treml M (2001) Regional dendritic and spine variation in human cerebral cortex: a quantitative Golgi study. Cereb Cortex 11:558-571.

Jacobs RE, Ahrens ET, Dickinson ME, Laidlaw D (1999) Towards a microMRI atlas of mouse development. Comput Med Imaging Graph 23:15-24.

Johnson GA, Benveniste H, Engelhardt RT, Qiu H, Hedlund LW (1997) Magnetic resonance microscopy in basic studies of brain structure and function. Ann NY Acad Sci 820:139-147.

Kostovic I, Rakic P (1990) Developmental history of the transient subplate zone in the visual and somatosensory cortex of the macaque monkey and human brain. J Comp Neurol 297:441-470.

Libersat F, Duch C (2004) Mechanisms of dendritic maturation. Mol Neurobiol 29:303-320.

Maas LC, Mukherjee P, Carballido-Gamio J, Veeraraghavan S, Miller SP, Partridge SC, Henry RG, Barkovich AJ, Vigneron DB (2004) Early laminar organization of the human cerebrum demonstrated with diffusion tensor imaging in extremely premature infants. NeuroImage 22:1134-1140 
McAllister AK (2000) Cellular and molecular mechanisms of dendrite growth. Cereb Cortex 10:963-973.

McKinstry RC, Mathur A, Miller JH, Ozcan A, Snyder AZ, Schefft GL, Almli CR, Shiran SI, Conturo TE, Neil JJ (2002) Radial organization of developing preterm human cerebral cortex revealed by non-invasive water diffusion anisotropy MRI. Cereb Cortex 12:1237-1243.

Miller M (1981) Maturation of rat visual cortex. I. A quantitative study of Golgi-impregnated pyramidal neurons. J Neurocytol 10:859-878.

Miller M, Peters A (1981) Maturation of rat visual cortex. II. A combined Golgi-electron microscope study of pyramidal neurons. J Comp Neurol 203:555-573.

Mori S, Itoh R, Zhang J, Kaufmann WE, van Zijl PCM, Solaiyappan M, Yarowsky P (2001) Diffusion tensor imaging of the developing mouse brain. Magn Reson Med 46:18-23.

Moseley ME, Cohen Y, Kucharczyk J, Mintorovitch J, Asgari HS, Wendland MF, Tsuruda J, Norman D (1990) Diffusion-weighted MR imaging of anisotropic water diffusion in cat central nervous system. Radiology 176:439-445.

Mukherjee P, Miller JH, Shimony JS, Philip JV, Nehra D, Snyder AZ, Conturo TE, Neil JJ, McKinstry RC (2002) Diffusion-tensor MR imaging of gray and white matter development during normal human brain maturation. Am J Neuroradiol 23:1445-1456.

Neil J, Shiran S, McKinstry R, Schefft G, Snyder A, Almli C, Akbudak E, Arnovitz J, Miller J, Lee B, Conturo T (1998) Normal brain in human newborns: apparent diffusion coefficient and diffusion anisotropy measured by using diffusion tensor MR imaging. Radiology 209:57-66.

Neil JJ, Miller J, Mukherjee P, Huppi PS (2002) Diffusion tensor imaging of normal and injured developing human brain-a technical review. NMR Biomed 15:543-552.

Partridge SC, Mukherjee P, Henry RG, Miller SP, Berman JI, Jin H, Lu Y, Glenn OA, Ferriero DM, Barkovich AJ, Vigneron DB (2004) Diffusion tensor imaging: serial quantitation of white matter tract maturity in premature newborns. NeuroImage 22:1302-1314.

Paxinos G, Watson C (2005) The rat brain in stereotaxic coordinates. Burlington, MA: Elsevier Academic Press.

Rakic P (2002) Evolving concepts of cortical radial and areal specification. Prog Brain Res 136:265-280.

Ramon-Moliner E (1962) An attempt at classifying nerve cells on the basis of their dendritic patterns. J Comp Neurol 119:211-227.

Ramon y Cajal S (1909) Histologie du systeme nervenux de l'homme dt des vertebras. Paris: Maloine.

Schneider JF, Il'yasov KA, Hennig J, Martin E (2004) Fast quantitative diffusion-tensor imaging of cerebral white matter from the neonatal period to adolescence. Neuroradiology 46:258-266.

Shaffer JP (1995) Multiple hypothesis testing. Annu Rev Psychol 46:561-584.
Sidman R, Rakic P, eds (1982) Development of the human central nervous system. Springfield, IL: Thomas.

Sidman RL, Rakic P (1973) Neuronal migration, with special reference to developing human brain: a review. Brain Res 62:1-35.

Sizonenko SV, Camm EJ, Garbow JR, Maier SE, Inder TE, Neil JJ, Huppi PS (2007) Developmental changes and injury induced disruption of the radial organization of the cortex in the immature rat brain revealed by in vivo diffusion tensor MRI. Cereb Cortex 17:2609-2617.

Song SK, Sun SW, Ramsbottom MJ, Chang C, Russell J, Cross AH (2002) Dysmyelination revealed through MRI as increased radial (but unchanged axial) diffusion of water. NeuroImage 17:1429-1436.

Song SK, Kim JH, Lin SJ, Brendza RP, Holtzman DM (2004) Diffusion tensor imaging detects age-dependent white matter changes in a transgenic mouse model with amyloid deposition. Neurobiol Dis 15:640-647.

Sun SW, Neil JJ, Song SK (2003) Relative indices of water diffusion anisotropy are equivalent to live and formalin-fixed mouse brains. Magn Reson Med 50:743-748.

Sun SW, Neil JJ, Liang HF, He YY, Schmidt RE, Hsu CY, Song SK (2005) Formalin fixation alters water diffusion coefficient magnitude but not anisotropy in infarcted brain. Magn Reson Med 53:1447-1451.

Thornton JS, Ordidge RJ, Penrice J, Cady EB, Amess PN, Punwani S, Clemence M, Wyatt JS (1997) Anisotropic water diffusion in white and gray matter on the neonatal piglet brain before and after transient hypoxiaischaemia. Magn Res Imaging 15:433-440.

Whitford KL, Dijkhuizen P, Polleux F, Ghosh A (2002) Molecular control of cortical dendrite development. Annu Rev Neurosci 25:127-149.

Wong RO, Ghosh A (2002) Activity-dependent regulation of dendritic growth and patterning. Nat Rev Neurosci 3:803-812.

Zhang J, van Zijl PC, Mori S (2002) Three dimensional diffusion tensor magnetic resonance micro-imaging of adult mouse brain and hippocampus. NeuroImage 15:892-901.

Zhang J, Richards LJ, Yarowsky P, Huang H, van Zijl PC, Mori S (2003) Three-dimensional anatomical characterization of the developing mouse brain by diffusion tensor microimaging. NeuroImage 20:1639-1648.

Zhang J, Chen YB, Hardwick JM, Miller MI, Plachez C, Richards LJ, Yarowsky P, van Zijl P, Mori S (2005) Magnetic resonance diffusion tensor microimaging reveals a role for Bcl-x in brain development and homeostasis. J Neurosci 25:1881-1888.

Zhang J, van Zijl PCM, Laterra J, Salhotra A, Lal B, Mori S, Zhou J (2007) Unique pattern of diffusion directionality in rat brain tumors revealed by high-resolution diffusion tensor MRI. Magn Reson Med 58:454-462.

Zhang ZW (2004) Maturation of layer V pyramidal neurons in the rat prefrontal cortex: intrinsic properties and synaptic function. J Neurophysiol 91:1171-1182. 